\title{
Dietary capsanthin, the main carotenoid in paprika (Capsicum annuum), alters plasma high-density lipoprotein-cholesterol levels and hepatic gene expression in rats
}

\author{
Koichi Aizawa* and Takahiro Inakuma \\ Corporate Planning Division, Research Institute, Kagome Co., Ltd, 17 Nishitomiyama, Nasushiobara-shi, Tochigi 329-2762, Japan
}

(Received 9 January 2009 - Revised 22 June 2009 - Accepted 23 June 2009 - First published online 31 July 2009)

The effects of dietary capsanthin, the main carotenoid in paprika (Capsicum annuum), on lipid metabolism were examined. Young male Wistar rats were fed diets containing paprika powder, paprika organic solvent extract, residue of paprika extract, and purified capsanthin. Administration of purified capsanthin for 2 weeks resulted in a significant increase in plasma HDL-cholesterol $(P<0 \cdot 05)$ without detectable differences in plasma total cholesterol and TAG concentrations. A statistically significant correlation $(r 0.567 ; P<0 \cdot 001)$ was found between dietary capsanthin concentrations and plasma HDL-cholesterol concentrations. Animals receiving diets containing two different capsanthin concentrations exhibited dose-dependent increases in plasma HDL-cholesterol $(r 0.597 ; P<0.005)$. While capsanthin was absent in the liver of animals fed the basal diet, it increased markedly in capsanthin-fed animals $(P<0 \cdot 001)$. Quantitative analyses of hepatic mRNA levels revealed that capsanthin administration resulted in up-regulation of mRNA for apoA5 and lecithin cholesterol acyltransferase (LCAT), without significant differences in other mRNA levels related to HDL-cholesterol metabolism. These results suggest that capsanthin had an HDL-cholesterol-raising effect on plasma, and the potential to increase cholesterol efflux to HDL particles by increasing apoA5 levels and/or enhancement of LCAT activity.

Paprika: Capsanthin: Lipid metabolism: Hepatic genes

CVD is one of the leading causes of morbidity and mortality worldwide. It is suggested that low levels of HDL-cholesterol constitute an independent risk factor for $\mathrm{CVD}^{(1-3)}$. Also, a recently concluded clinical intervention trial supports the idea that increasing the levels of HDLcholesterol can protect against clinical $\mathrm{CVD}^{(4)}$. Based on these observations and current concepts regarding the antiatherogenic roles of HDL in promoting reverse cholesterol transport $^{(5)}$ and as an antioxidant ${ }^{(6)}$, interventions involving an increase in HDL concentrations are expected to constitute an important therapeutic option for CVD prevention. For example, consumption of polyphenol-rich fruit juice or cocoa chocolate has been shown to increase plasma HDL-cholesterol concentrations ${ }^{(7-9)}$. Additionally, dietary supplementation with concentrated tomato juice, which has a high antioxidant capacity due to its high content of lycopene, significantly increased lycopene and HDLcholesterol levels $^{(10)}$.

Ripe paprika fruit (Capsicum annuum) is widely used as a vegetable and food additive, as this fruit is considered to be a good source of carotenoid pigments. Capsanthin is the major carotenoid present in paprika, and is present in an acylated form with fatty acids ${ }^{(11,12)}$. This carotenoid, which does not possess provitamin A activity, has been shown to be effective as a free-radical scavenger ${ }^{(13)}$.

Oshima et al. ${ }^{(14)}$ studied the accumulation and clearance of capsanthin in the plasma of human males after ingestion of paprika juice. These studies revealed that dietary capsanthin was absorbed into the body and distributed to plasma lipoproteins. Furthermore, it was confirmed that xanthophylls, including capsanthin, are distributed to HDL in larger amounts than to LDL, when compared with hydrocarbon carotenoids. Xanthophylls can act as antioxidants against free radical attack and exposure to singlet oxygen in plasma lipoproteins ${ }^{(15,16)}$. Therefore, dietary xanthophylls seem to participate in the primary defence mechanism of HDL against oxidative stress, and may also be expected to affect lipid metabolism and/or maintain favourable blood lipid profiles. In fact, some reports have suggested that certain carotenoids may affect HDLcholesterol concentrations ${ }^{(17,18)}$ and alter adipocytokine levels $^{(19,20)}$ or hepatic gene expression ${ }^{(21)}$. Therefore, it is reasonable to speculate that ingestion of paprika, which possesses abundant capsanthin, may modulate lipid metabolism.

The purpose of the present study was to evaluate the effect of capsanthin, the main carotenoid in paprika, on in vivo lipid metabolism in rats.

Abbreviations: ABC, ATP-binding cassette transporter; BD, basal diet; CAP, basal diet with purified capsanthin; EXT, basal diet with paprika extract; HIGH, highcapsanthin diet; LCAT, lecithin cholesterol acyltransferase; LOW, low-capsanthin diet; LPL, lipoprotein lipase; PAP, basal diet with paprika powder; RES, basal diet with residue of extract; SR-B1, scavenger receptor class B type 1.

* Corresponding author: Dr Koichi Aizawa, fax +81 28739 1038, email Koichi_Aizawa@kagome.co.jp 


\section{Materials and methods}

\section{Animals}

Known-pathogen-free male Crlj:WI rats (Wistar rats, aged 4 weeks) were purchased from Charles River Laboratories Japan, Inc. (Yokohama, Japan) and maintained on a normal CE-2 (CLEA, Tokyo, Japan) diet for 1 week before starting the experiment. All rats were housed individually in stainlesssteel cages under controlled conditions (temperature $23 \pm 1{ }^{\circ} \mathrm{C}$, humidity $55 \pm 5 \%$, lights on from 07.00 to 19.00 hours). At the end of the experiments, the rats were anaesthetised with sodium pentobarbital and blood and liver samples were taken. Plasma samples were stored at $-80^{\circ} \mathrm{C}$ until analysed. Livers were immediately frozen and kept at $-80^{\circ} \mathrm{C}$ until analysed. All animals were treated in accordance with guidelines established by the Japanese Society of Nutrition and Food Science (Law 105 and Notification 6 of the Japanese government). The experimental protocol was approved by the Kagome Animal Use Committee.

\section{Diets and preparation of samples}

The basic diet contained the following ingredients (per $\mathrm{kg}$ ): $250 \mathrm{~g}$ casein, $453 \mathrm{~g}$ maize starch, $200 \mathrm{~g}$ sucrose, $50 \mathrm{~g}$ maize oil, $35 \mathrm{~g}$ American Institute of Nutrition (AIN)-93 mineral mix, $10 \mathrm{~g}$ AIN-93 vitamin mixture and $2 \mathrm{~g}$ choline chloride. The diet ingredients were purchased from Oriental Yeast Co. (Tokyo, Japan). The composition of the other experimental diets resembled the basic diet, except that paprika powder, paprika extract, residue of paprika extract or purified capsanthin replaced an equivalent weight of maize starch.

Paprika powder was obtained by lyophilising paprika paste (TAT, Istanbul, Turkey). Paprika extract and its residue were prepared by organic solvent extraction. Briefly, paprika powder $(20 \mathrm{~g})$ was extracted by hexane-acetone-ethanoltoluene (10:7:6:7, by vol.) three times to separate the soluble organic components, including carotenoids ${ }^{(22)}$. The extracts were combined and evaporated to produce the paprika extract $(0.5 \mathrm{~g})$. In addition, paprika extract residue (approximately $19.5 \mathrm{~g}$ ) was acquired by the removal of organic solvents from the extraction residue. Capsanthin concentrations in the paprika powder, paprika extract and residue of paprika extract were determined by $\operatorname{HPLC}^{(23)}$ at $2.43,64.97$ and $0.62 \mathrm{mmol} / \mathrm{kg}$, respectively.

Purified capsanthin for animal feed was extracted from commercially available non-acylated free capsanthin-containing powder (Capsanthal; BASF Japan Ltd, Tokyo, Japan), and purified by a chromatographic method. Briefly, Capsanthal was extracted by methanol and the extracts were applied to preparative HPLC (GL Science Inc., Tokyo, Japan) with a Soken pack octadecylsilyl (ODS-ST-C) column (Soken Chemical \& Engineering Co., Ltd, Tokyo, Japan) and eluted with methanol. All fractions containing capsanthin were collected and evaporated. Crude capsanthin extracts were subjected to silica gel column chromatography (Wako-gel C-200; Wako, Tokyo, Japan) and sequential elutions of a solvent mixture of dichloromethane and methanol. The fractions containing capsanthin were pooled, and the solvent was removed in vacuo to obtain purified capsanthin. Capsanthin purity $(>90 \%)$ was determined by absorption spectroscopy and HPLC methodology ${ }^{(23)}$.

\section{Experimental design}

In the present study, the main objective was to examine the effect of paprika or capsanthin ingestion on lipid metabolism. The feeding period of 2 weeks was determined by previous reports, which showed remarkable changes in lipid profiles and hepatic gene expression after ingestion of food ingredients ${ }^{(24-26)}$. Furthermore, the maximum dose of capsanthin $(0.49 \mathrm{mmol} / \mathrm{kg}$ diet $)$ was chosen to produce an appreciable accumulation in the liver, according to previous experiments $^{(27,28)}$.

In experiment 1 , after a maintenance period of 1 week, rats were divided into five groups of six animals each, with similar average body weights. The control group was fed a basal diet (BD), and the experimental groups were fed a basal diet with paprika powder (PAP), paprika extract (EXT), residue of paprika extract (RES) or purified capsanthin (CAP), as shown in Table 1 . The diets and water were given ad libitum for 2 weeks. Faeces were collected and measured for $3 \mathrm{~d}$ at the end of the experiment.

In experiment 2, rats were divided into three groups of eight animals. The groups were fed either a basal diet (control) or a basal diet supplemented with one of two different capsanthin concentrations (low-capsanthin diet (LOW), 0.16g purified capsanthin/kg diet; high-capsanthin diet (HIGH), 0.32 g purified capsanthin $/ \mathrm{kg}$ diet). The diet capsanthin concentrations were 0.25 and $0.49 \mathrm{mmol} / \mathrm{kg}$ diet, respectively. The diets and water were provided ad libitum for 2 weeks. The concentration of liver capsanthin and relative mRNA concentrations of hepatic genes were measured in this experiment.

All blood samples were obtained from non-fasted rats. The concentrations of plasma total cholesterol, HDL-cholesterol and TAG were measured manually using commercial in vitro enzymic test kits (Wako, Tokyo, Japan).

The previously described method ${ }^{(16)}$ was modified and used for measuring carotenoids in liver. Briefly, the tissue was homogenised and saponified by the addition of $60 \% \mathrm{KOH}$ and $3 \%$ butyrated hydroxytoluene in ethanol, heated at $40^{\circ} \mathrm{C}$ for $30 \mathrm{~min}$, and subsequently extracted (twice) with hexanedichloromethane $(4: 1, \mathrm{v} / \mathrm{v})$. The supernatant fraction was dried and reconstituted in a hexane-acetone-ethanol-toluene

Table 1. Composition of experimental diets in experiment 1 (g/kg diet)

\begin{tabular}{lrrrrc}
\hline & \multicolumn{5}{c}{ Group } \\
\cline { 2 - 6 } Ingredients & BD & PAP & EXT & RES & CAP \\
\hline Casein (g) & 250 & 250 & 250 & 250 & 250 \\
Maize starch (g) & 453 & 253 & 448 & 258 & $452 \cdot 68$ \\
Sucrose (g) & 200 & 200 & 200 & 200 & 200 \\
Maize oil (g) & 50 & 50 & 50 & 50 & 50 \\
AIN-93 mineral mixture (g) & 35 & 35 & 35 & 35 & 35 \\
AIN-93 vitamin mixture (g) & 10 & 10 & 10 & 10 & 10 \\
Choline chloride (g) & 2 & 2 & 2 & 2 & 2 \\
Paprika powder (g) & - & 200 & - & - & - \\
Paprika extract (g) & - & - & 5 & - & - \\
Residue of paprika extract (g) & - & - & - & 195 & - \\
Purified capsanthin (g) & - & - & - & - & 0.32 \\
\hline
\end{tabular}

$\mathrm{BD}$, basal diet (capsanthin concentration $0.00 \mathrm{mmol} / \mathrm{kg}$ diet); PAP, basal diet with paprika powder $(0.49 \mathrm{mmol} / \mathrm{kg}$ diet); EXT, basal diet with paprika extract $(0.32 \mathrm{mmol} / \mathrm{kg}$ diet $)$; RES, basal diet with residue of extract $(0.12 \mathrm{mmol} / \mathrm{kg}$ diet $)$; CAP, basal diet with purified capsanthin $(0.49 \mathrm{mmol} / \mathrm{kg}$ diet $)$; AIN, American Institute of Nutrition. 
solvent. Analyses were performed using a Shimadzu SPD-M10 $v p$ diode array detector (Shimadzu, Kyoto, Japan) and C30 carotenoid column $(5 \mu \mathrm{m}, 250 \times 4.6 \mathrm{~mm}$; YMC, Wilmington, NC, USA) with a flow rate of $1.0 \mathrm{ml} / \mathrm{min}$.

Experimental diets were stored at $-30^{\circ} \mathrm{C}$ until required, with fresh food provided daily. Food intake was measured daily, and body weight and water intake were measured three times per week.

\section{Real-time quantitative PCR analysis of gene expression}

Hepatic gene expression (relative mRNA concentrations) was measured in the rats from experiment 2 . For analysis of gene expression, total RNA was extracted from rat liver samples using Trizol reagent (Invitrogen, Tokyo, Japan) according to the manufacturer's instructions. RNA was spectrophotometrically quantified $\left(\mathrm{A}_{260}\right)$ and its integrity verified by agarose gel electrophoresis using ethidium bromide for visualisation. Total RNA was reverse transcribed with a PrimeScript RT reagent kit (Takara Bio Inc., Shiga, Japan) for cDNA synthesis. The relative mRNA quantities of ATP-binding cassette transporter A1 (ABC-A1), apoA1, apoA5, apoC3, hepatic lipase, lecithin cholesterol acyltransferase (LCAT), lipoprotein lipase (LPL) and scavenger receptor class B type 1 (SR-B1) were measured by real-time quantitative PCR using SYBR Green I (Takara Bio Inc.) and the ABI PRISM 7000 Sequence Detection System (Applied Biosystems Japan Ltd, Tokyo, Japan). Real-time quantitative PCR was performed using a ninety-six-well PCR plate and a reaction mixture $(50 \mu \mathrm{l})$ containing $25 \mu \mathrm{l} \mathrm{SYBR}$ Premix Ex Taq ${ }^{\mathrm{TM}}$ II $(2 \times), 1 \mu l$ ROX Reference Dye $(10 \times), 16 \mu l$ Rnase-free water (all from Takara Bio Inc.), $2 \mu$ l forward primer $(10 \mu \mathrm{M}), 2 \mu \mathrm{l}$ reverse primer $(10 \mu \mathrm{M})$ and $4 \mu \mathrm{l}$ template. The primer oligonucleotides were selected using the online primerselect system (Takara Bio Inc.) and Primerpairs software (Applied Biosystems Japan Ltd) from database sequences. The primer sequences used for RT-PCR were as follows: 5'-CAG CAA CTA CAG TGG CGG TAA CA-3' (forward) and $5^{\prime}$-AAT GCT TAG GGC ACA ATT CCA CA-3' (reverse) for rat ABC-A1 (NM_178095); 5'-ATC TAA AGG TTG TGG CCG AGG A-3' (forward) and 5'-CTC GAT CAG GGT AGG GTG GTT C-3' (reverse) for rat apoA1 (NM_012738); 5'-GGA GTG TGT ACA TCC CTG CCA GT-3' (forward) and $5^{\prime}$-ACT GCA GAG GGC TCA GTT CCT TAT T-3' (reverse) for rat apoA5 (NM_080576); 5'-ATC CTT GCT GCT GGG CTC TAT G-3' (forward) and 5'-TTC AGG GAT TTG AAG CGA TTG TC-3' (reverse) for rat apoC3 (NM_012501); 5'-GGC ACA GTC AAG GCT GAG AAT G-3' (forward) and 5'-ATG GTG GTG AAG ACG CCA GTA-3' (reverse) for rat glyceraldehyde-3-phosphate dehydrogenase (GAPDH; NM_017008); 5'-ACC CGG AAA CAC TGC AGG AG-3' (forward) and 5'-GTT GGG ACT GTC GGG ACT TCA-3' (reverse) for rat hepatic lipase (NM_012597); 5'-CCC AAG GCT GAA CTC AGT AAC CA-3' (forward) and $5^{\prime}$-CGG TAG CAC AGC CAG TTT ACC A-3' (reverse) for rat LCAT (NM_017024); 5'-CCA ATC GTT AGC ATT TCG TTT GAG-3' (forward) and 5'-TTG CGC AGT GCA GAA TTT GA-3' (reverse) for rat LPL (NM_012598); 5'-GTT CCG TGA AGA TGC AGC TGA G-3 ${ }^{\prime}$ (forward) and $5^{\prime}$-AAC CAC AGC AAT GGC AGG ACT AC-3' (reverse) for rat SR-B1 (NM_031541).
For determination of mRNA concentration, a threshold cycle $(C \mathrm{t})$ and amplification efficiency were obtained from each amplification curve using the Applied Biosystems software 1.1 (Applied Biosystems Japan Ltd). The expression signal of the housekeeping gene GAPDH served as an internal control for normalisation.

\section{Statistical analysis}

Results are expressed as mean values and standard deviations for six or eight rats. Data were analysed by one-way ANOVA. If significance was observed, post hoc pairwise comparisons were conducted using Tukey's test. The correlations were investigated by Pearson's coefficient test. All the analyses were performed with the SPSS 15.0J software computerised statistical analysis program (SPSS Japan Inc., Tokyo, Japan). $P<0.05$ was considered statistically significant.

\section{Results \\ Effects of paprika extracts on body weight and plasma lipids in rats (experiment 1)}

Diets containing different paprika fractions, paprika powder (PAP), paprika extract (EXT), residue of paprika extract (RES) and purified capsanthin (CAP), were examined in this experiment. There were no noticeable differences in bodyweight gain, food intake and liver weight among the groups (Table 2). The wet faecal weights in the PAP and RES groups were significantly $(P<0.05)$ higher than those in the basal diet (BD), EXT and CAP groups. There were no detectable differences in plasma total cholesterol and TAG concentrations. However, the HDL-cholesterol concentration in the CAP group was significantly $(P<0.05)$ higher than that in the BD group. Significant differences in HDLcholesterol concentration were not observed between the PAP, EXT and RES groups, and the BD and CAP groups. However, using Pearson's correlation analysis, a statistically significant correlation $(r 0.567 ; P<0.001)$ was found between the capsanthin concentration in the diet and the plasma HDL-cholesterol concentration.

Effect of capsanthin on body weight, plasma lipid and hepatic mRNA levels (experiment 2)

Two different concentrations of capsanthin were compared in this experiment. HDL-cholesterol levels tended to increase (17\% increase) when capsanthin was administered at a low dose (LOW group) and were significantly raised when administered at a high dose (HIGH group; $P<0 \cdot 01 ; 30 \%$ increase). No significant differences were observed in body-weight gain, food intake, liver weight, and plasma total cholesterol and TAG concentrations among the groups (Table 3 ). There was no capsanthin content in the liver of animals fed the $\mathrm{BD}$, but it was increased markedly with the administration of capsanthin-containing diets $(P<0.001$ between each group). Statistically significant relationships were observed between plasma HDL-cholesterol concentrations and capsanthin concentrations in the diet $(r 0.597 ; P<0.005)$ or liver $(r 0.583 ; P<0.005)$ (Fig. 1). 
Table 2. Body weight, food intake, liver weight, faecal weight and plasma lipid contents in rats fed basal and experiment 1 diets for 2 weeks (Mean values and standard deviations for six rats per group)

\begin{tabular}{|c|c|c|c|c|c|c|c|c|c|c|}
\hline & \multicolumn{2}{|c|}{$\mathrm{BD}$} & \multicolumn{2}{|c|}{ PAP } & \multicolumn{2}{|c|}{ EXT } & \multicolumn{2}{|c|}{ RES } & \multicolumn{2}{|c|}{ CAP } \\
\hline & Mean & SD & Mean & SD & Mean & SD & Mean & SD & Mean & SD \\
\hline Body weight $(\mathrm{g})^{\star}$ & 241.90 & $12 \cdot 26$ & 237.85 & 18.91 & 238.84 & $15 \cdot 01$ & 241.70 & $9 \cdot 69$ & 235.78 & 11.86 \\
\hline Body-weight gain (g) & 107.48 & $5 \cdot 34$ & $103 \cdot 25$ & $13 \cdot 65$ & $104 \cdot 66$ & $10 \cdot 03$ & 107.59 & $8 \cdot 32$ & 101.77 & $8 \cdot 13$ \\
\hline Food intake $(\mathrm{g} / \mathrm{d})$ & $19 \cdot 14$ & 0.68 & 18.39 & 1.68 & 18.48 & 0.90 & $19 \cdot 74$ & 1.32 & 18.66 & 1.04 \\
\hline Faecal weight (g per $3 \mathrm{~d}) \dagger$ & $1.36^{\mathrm{b}}$ & 0.17 & $2 \cdot 74^{\mathrm{a}}$ & 0.43 & $1.56^{\mathrm{b}}$ & 0.21 & $2 \cdot 89^{\mathrm{a}}$ & 0.84 & $1.37^{\mathrm{b}}$ & 0.21 \\
\hline Liver weight (g) & 7.74 & 0.73 & $7 \cdot 84$ & 1.00 & $7 \cdot 72$ & 0.50 & $7 \cdot 80$ & 0.56 & $7 \cdot 70$ & 0.87 \\
\hline Plasma total cholesterol $(\mathrm{mmol} / \mathrm{l})$ & 1.86 & 0.55 & 2.42 & 0.43 & $2 \cdot 40$ & 0.19 & $2 \cdot 13$ & 0.41 & 1.85 & 0.25 \\
\hline Plasma HDL-cholesterol ( $\mathrm{mmol} / \mathrm{l})$ & $1 \cdot 22^{\mathrm{b}}$ & 0.24 & $1.61^{a, b}$ & 0.28 & $1.50^{\mathrm{a}, \mathrm{b}}$ & 0.12 & $1 \cdot 37^{a, b}$ & 0.43 & $1.76^{\mathrm{a}}$ & 0.25 \\
\hline Plasma TAG $(\mathrm{mmol} / \mathrm{l})$ & 0.96 & 0.43 & 1.02 & 0.57 & 1.03 & 0.16 & 0.76 & 0.26 & 0.90 & 0.48 \\
\hline
\end{tabular}

$\mathrm{BD}$, basal diet; PAP, basal diet with paprika powder; EXT, basal diet with paprika extract; RES, basal diet with residue of extract; CAP, basal diet with purified capsanthin.

${ }^{a, b}$ Mean values within a row with unlike superscript letters were significantly different $(P<0.05)$.

* Body weight at the end of the 2-week feeding period.

+ Wet faecal weight for $3 \mathrm{~d}$ at the end of the 2-week feeding period.

Real-time quantitative PCR analyses were performed to measure mRNA levels in rat liver from the three dietary groups (Fig. 2). Administration of capsanthin caused a significant increase $(P<0.05)$ in the relative quantity of apoA5 mRNA levels in LOW and HIGH groups. Those levels were positively correlated with the capsanthin concentration in liver $(r 0.514 ; P<0.01)$. Also, the relative quantity of LCAT mRNA in the HIGH group was significantly higher than that of the BD group. No significant differences or correlations were observed for relative quantities of other mRNA levels related to HDL-cholesterol metabolism in this experiment.

\section{Discussion}

Previously, preliminary experiments revealed that administration of paprika increased plasma HDL-cholesterol in rats (K Aizawa and T Inakuma, unpublished results). The present study was conducted to examine what constituent of paprika (C. аппиит) principally affected lipid metabolism in vivo in rats, hypothesising that capsanthin, the main carotenoid in paprika, might be responsible. As shown in Table 2, administration of purified capsanthin significantly increased $(P<0.05$; $44 \%$ increase) plasma HDL-cholesterol levels. Furthermore, administration of two different capsanthin concentrations resulted in a dose-dependent increase in plasma HDLcholesterol in experiment 2 (Table 3). Consequently, we concluded that the main active component was capsanthin. This conclusion was supported by the results from experiment 1 , which showed that a significant correlation ( $r$ 0.567; $P<0.001)$ was found between diet capsanthin concentrations and plasma HDL-cholesterol concentrations.

In experiment 1 , it was observed that faecal weight in the PAP and RES groups was significantly higher $(P<0.05 ; 202$ and $213 \%$ increase compared with the BD group, respectively) than that of the BD, EXT and CAP groups. These effects were attributed to the dietary fibre component of the experimental diets. It is reported that the administration of dietary fibre results in a dose-dependent increase in faecal weight $^{(29,30)}$. It was estimated that paprika powder (PAP) and residue of paprika extract (RES) are composed of 51.8 and $53.1 \%$ carbohydrate (including dietary fibre), respectively. These factors raised the dietary fibre content by approximately $10 \%$ in PAP and RES experimental diets. Additionally, it has been indicated that certain kinds of dietary fibre influence total cholesterol or TAG levels in blood, and increase bile acid or neutral sterol excretions ${ }^{(31,32)}$. In the present study, we did not delve deeper into the effect of dietary fibre, because we were particularly intrigued by the ability

Table 3. Body weight, food intake, liver weight, plasma lipid contents and liver capsanthin concentration in rats fed basal and experiment 2 diets for 2 weeks

(Mean values and standard deviations for eight rats per group)

\begin{tabular}{|c|c|c|c|c|c|c|}
\hline & \multicolumn{2}{|c|}{ BD } & \multicolumn{2}{|c|}{ LOW } & \multicolumn{2}{|c|}{$\mathrm{HIGH}$} \\
\hline & Mean & SD & Mean & SD & Mean & SD \\
\hline Body weight $(g)^{\star}$ & $265 \cdot 88$ & $13 \cdot 82$ & 261.95 & $16 \cdot 91$ & $272 \cdot 30$ & $16 \cdot 18$ \\
\hline Body-weight gain (g) & $103 \cdot 81$ & 7.98 & 101.03 & $12 \cdot 46$ & $109 \cdot 29$ & 11.45 \\
\hline Food intake $(\mathrm{g} / \mathrm{d})$ & 23.74 & 0.77 & 23.08 & 1.55 & 23.82 & 1.24 \\
\hline Liver weight $(\mathrm{g})$ & $7 \cdot 32$ & 0.54 & $7 \cdot 36$ & 1.07 & $8 \cdot 14$ & 0.91 \\
\hline Plasma total cholesterol (mmol/l) & 1.91 & 0.37 & 1.91 & 0.25 & 2.03 & 0.50 \\
\hline Plasma HDL-cholesterol (mmol/l) & $1.39^{\mathrm{b}}$ & 0.22 & $1.63^{a, b}$ & 0.23 & $1.81^{\mathrm{a}}$ & 0.29 \\
\hline Plasma TAG (mmol/l) & 0.81 & $0 \cdot 12$ & 0.95 & 0.37 & 1.02 & 0.21 \\
\hline Liver capsanthin (mmol/mg wet tissue) & $0.00^{\mathrm{a}}$ & 0.00 & $0.22^{\mathrm{b}}$ & 0.05 & $0.40^{\mathrm{C}}$ & 0.14 \\
\hline
\end{tabular}

$\mathrm{BD}$, basal diet; LOW, basal diet with purified capsanthin $(0.16 \mathrm{~g} / \mathrm{kg}$ diet $) ; \mathrm{HIGH}$, basal diet with purified capsanthin $(0.32 \mathrm{~g} / \mathrm{kg}$ diet).

${ }_{a, b, c}$ Mean values within a row with unlike superscript letters were significantly different $(P<0.05)$

${ }^{*}$ Body weight at the end of the 2-week feeding period. 


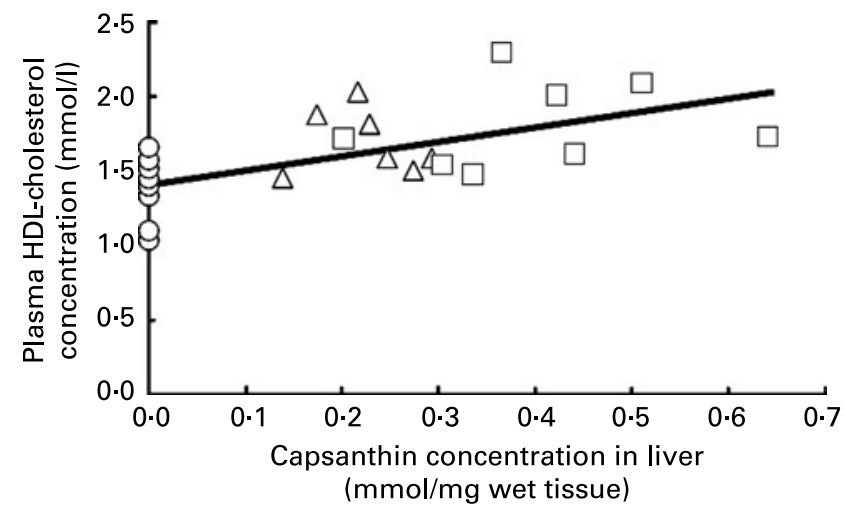

Fig. 1. Correlation ( $r 0.583 ; P<0.005)$ between plasma HDL-cholesterol concentration and capsanthin concentration in the liver of rats fed the basal diet $(O)$, the low capsanthin-containing diet $(\triangle)$ and the high capsanthincontaining diet $(\square)$ for 2 weeks.

of capsanthin to increase HDL-cholesterol levels. The cholesterol-lowering effects of dietary fibre are primarily observed in the LDL portion, and dietary fibre did not produce substantial changes in HDL-cholesterol levels ${ }^{(33)}$. We may be able to observe blood lipid changes induced by the addition of paprika-derived dietary fibre in the experimental diet if the experimental duration is extended.

Quantitative analysis of liver mRNA in the capsanthinadministered group showed a significant increase in apoA5 and LCAT expression, without notable changes in ABC-A1, apoA1, apoC3, hepatic lipase, LPL, and SR-B1 (Fig. 2). HDL is synthesised through a complex pathway ${ }^{(34)}$. HDL assembly initially involves cell surface ABC-A1 transporter-mediated transfer of phospholipids and cholesterol to extracellular lipid-poor apoA1. This is followed by remodelling of the plasma compartment of HDL particles, by the esterification of cholesterol by the enzyme LCAT, the exchange between HDL and other lipoproteins of both apolipoproteins (apoA1 and other less abundant apolipoproteins) and lipids, and the putative transfer of additional cellular cholesterol to the growing particles by $\mathrm{SR}-\mathrm{B} 1^{(35)}$. Finally, HDL lipid hydrolysis is mediated by various lipases

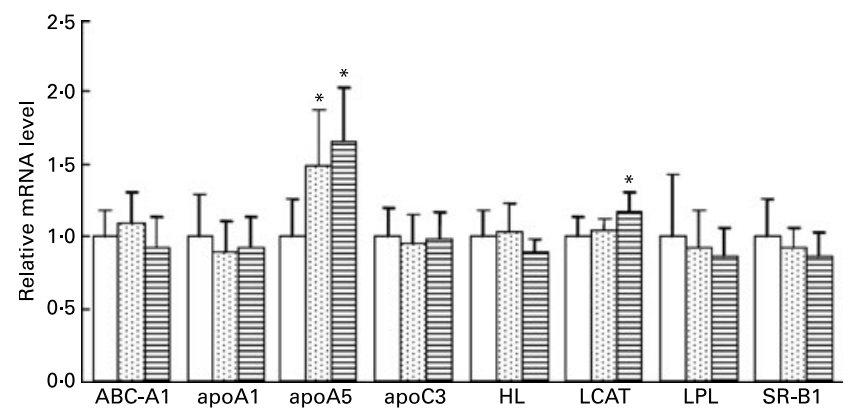

Fig. 2. Quantitative analysis of liver mRNA of rats fed the basal diet (BD; $\square$ ), the low capsanthin-containing diet (回) or the high capsanthin-containing diet (目) for 2 weeks. For each gene, the mRNA level is shown relative to its level in the BD group (set at 1.0). ABC, ATP-binding cassette transporter; HL, hepatic lipase; LCAT, lecithin cholesterol acyltransferese; LPL, lipoprotein lipase; SR-B1, scavenger receptor class B type 1. Values are means for eight rats per group, with standard deviations represented by vertical bars. * Mean value was significantly different from that of the BD group $(P<0.05)$.
(LPL, hepatic lipase and endothelial lipase) and exchange of lipids by the cholesteryl ester transfer protein and by the phospholipid transfer protein. The increase in LCAT and apoA1 gene expression would normally contribute to an increase in HDL-cholesterol concentrations. In the present study, the hepatic apoA1 mRNA level among all groups was not significantly different, but LCAT mRNA was significantly increased by capsanthin administration. Therefore, one reason for the higher concentration of HDL-cholesterol in rats fed capsanthin diets could be due to alterations in the expression and/or activity of LCAT.

The role that LCAT plays in HDL metabolism has been established in both patients and animals with LCAT deficiency, as well as in animals over-expressing human LCAT. LCAT deficiency is associated with severely reduced concentrations of HDL, whereas transgenic animals overexpressing LCAT show markedly higher plasma HDL levels ${ }^{(36-38)}$. However, a number of studies point to a relationship between LCAT and apoA1 expression ${ }^{(39,40)}$.

The reason for the discrepancy in the present study that hepatic apoA1 mRNA levels were not correlated with LCAT mRNA levels is unclear. On the other hand, although the plasma apoA1 concentration was drastically reduced in LCAT knockout mice, there was no reduction in hepatic apoA1 mRNA ${ }^{(41)}$, suggesting that both genes may not be regulated in a coordinated manner. At present, the molecular mechanisms regulating LCAT are not well understood. However, capsanthin may only regulate LCAT mRNA expression, without affecting apoA1 mRNA expression.

Additionally, a significant increase in hepatic apoA5 mRNA levels was observed by administration of capsanthin (Fig. 2). ApoA5 is a newly discovered apolipoprotein, which was identified independently by two groups ${ }^{(42,43)}$. Disruption of the apoA5 gene in mice resulted in hypertriacylglycerolaemia, whereas overexpression led to decreased plasma TAG concentrations, thus establishing an important role for this protein in TAG homeostasis ${ }^{(44,45)}$. Marçais et al. ${ }^{(46)}$ reported that a mutation in the apoA5 gene led to severe hypertriacylglycerolaemia by exerting a dominant-negative effect on the plasma lipolytic system for TAG-rich lipoproteins. They suggested that apoA5 accelerated lipolysis by facilitating the interaction of TAG-rich lipoproteins with heparin sulfate-proteoglycanbound LPL. In the present study, plasma TAG levels did not change appreciably, in spite of the increase in the apoA5 mRNA level. One possibility is that the LPL mRNA level did not change (Fig. 2), indicating that LPL activity was not enhanced by administration of capsanthin. Also, only a small amount of capsanthin was supplemented into the normal diet for a short period of time ( 2 weeks). Alteration of the quantity and duration of capsanthin administration may clarify these remarkable observations.

$\mathrm{Qu}$ et al. ${ }^{(47)}$ indicated that apoA5 exerted an effect on HDL-cholesterol metabolism in APOC3 transgenic mice. They reported that increased apoA5 production promoted $\alpha$-HDL formation, resulting in significant increases in both the number and size of HDL particles. In addition, increased apoA5 levels were associated with enhanced LCAT activity. It appears that HDL particles with increased apoA5 content were associated with increased cholesterol-loading capacity. This hypothesis is consistent with the present results. Capsanthin may be thought to act in a similar manner, 
increasing cholesterol efflux to HDL particles by increasing apoA5 levels and/or enhancement of LCAT activity, thereby resulting in significant increases in plasma HDL-cholesterol.

In order to test this possibility in human subjects in an exploratory open study, we administered $320 \mathrm{~g}$ paprika juice (made from paprika paste (TAT, Istanbul, Turkey) diluted with water), containing $38.9 \mu \mathrm{mol}$ capsanthin per $\mathrm{d}$ for 2 weeks to nine healthy males. This treatment resulted in a significant $(P<0.05)$ increase $(26 \%)$ in plasma HDL-cholesterol concentrations (from 1.63 (SD 0.10) mmol/l before paprika juice supplementation to 2.05 (SD 0.15 ) $\mathrm{mmol} / \mathrm{l}$ after 2 weeks of consumption). There was a concomitant significant increase in plasma capsanthin levels (from 0.008 (SD 0.001) to $0.136(\mathrm{SD} 0.090) \mu \mathrm{mol} / \mathrm{l} ; \quad P<0.0005)$ and a tendency towards increased LCAT activity (from 18.86 (SD 10.37) to 26.48 (SD 8.10) $\mathrm{U} ; P=0.086$ ). In addition, there were no detectable differences in cholesteryl ester transfer protein levels and other lipid levels during the experiment (data not shown). This study was conducted as a pilot study by using a small number of subjects without a control group. Further, it is not feasible to transpose doses used in animal studies for use in human studies, because the bioavailability of carotenoids is distinctly different among species. Therefore, it is difficult to predict effective dosages due to species differences. However, the results are encouraging and justify further, more elaborate studies. Notably, these results do not contradict the results of the animal studies presented here, and they provide some indication as to the possible expectation that human subjects would exhibit responses similar to those in rats.

It has not been established whether other carotenoids in food, such as lycopene, $\alpha$-carotene, $\beta$-carotene, lutein and zeaxanthin, have the same effect as capsanthin. A number of reports have shown that blood concentrations of certain carotenoids were significantly correlated to HDL-cholesterol level $^{(17,18)}$. However, this same report ${ }^{(17)}$ and another recent report ${ }^{(48)}$ indicated that dietary carotenoid intake was not associated with HDL-cholesterol level. Interestingly, Waters et al. ${ }^{(49)}$ reported that there was a positive correlation between HDL size and plasma lutein level. This finding suggests that lutein may affect the construction or function of HDL. Furthermore, xanthophylls, including capsanthin and lutein, are distributed to HDL in larger amounts than to $\mathrm{LDL}^{(14)}$. Thus, it is possible that not only capsanthin, but also other xanthophylls, might affect plasma HDL-cholesterol level.

In conclusion, the present study shows that capsanthin, the main carotenoid in paprika, has a plasma HDL-cholesterolraising effect accompanied by a significant increase in hepatic apoA5 and LCAT mRNA. Additionally, we think that it is normal and reasonable that the small amount of capsanthin added to a normal diet, administered for a short period in rats, would not produce drastic changes in plasma lipid profiles and hepatic mRNA levels. Meanwhile, it is interesting and meaningful to be able to report that dietary intake of paprika can improve plasma lipid profiles.

\section{Acknowledgements}

The present study was partially supported by the New Food Creation Technology Research Association, a project of the Ministry of Agriculture, Forestry and Fisheries of Japan.
K. A. designed all experiments, carried out the main experimental work and wrote the manuscript. T. I. supported the idea of the manuscript and, as the director of the Biogenics Research Department, provided a number of suggestions. The authors also wish to thank the staff of the Biogenics Research Department, Kagome Co., Ltd, for their help with sample and experiment preparation.

None of the authors had any personal or financial conflict of interest.

\section{References}

1. Miller NE (1987) Associations of high-density lipoprotein subclasses and apolipoproteins with ischemic heart disease and coronary atherosclerosis. Am Heart J 113, 589-597.

2. Gordon DJ, Probstfield JL, Garrison RJ, et al. (1989) Highdensity lipoprotein cholesterol and cardiovascular disease. Four prospective American studies. Circulation 79, 8-15.

3. Castelli WP, Anderson K, Wilson PW, et al. (1992) Lipids and risk of coronary heart disease: the Framingham Study. Ann Epidemiol 2, 23-28.

4. Rubins HB, Robins SJ, Collins D, et al. (1999) Gemfibrozil for the secondary prevention of coronary heart disease in men with low levels of high-density lipoprotein cholesterol. Veterans Affairs High-Density Lipoprotein Cholesterol Intervention Trial Study Group. N Engl J Med 341, 410-418.

5. Tall AR (1990) Plasma high-density lipoproteins: metabolism and relationship to atherogenesis. J Clin Invest 86, 379-384.

6. Parthasarathy S, Barnett J \& Fong LG (1990) High-density lipoprotein inhibits the oxidative modification of low-density lipoprotein. Biochim Biophys Acta 1044, 275-283.

7. Kurowska EM, Spence JD, Jordan J, et al. (2000) HDLcholesterol-raising effect of orange juice in subject with hypercholesterolemia. Am J Clin Nutr 72, 1095-1100.

8. Albers AR, Varghese S, Vitseva O, et al. (2004) The anti-inflammatory effects of purple grape juice consumption in subjects with stable coronary artery disease. Arterioscler Thromb Vasc Biol 24, e179-e180.

9. Mursu J, Voutilainen S, Nurmi T, et al. (2004) Dark chocolate consumption increase HDL cholesterol conventration and chocolate fatty acid may inhibit lipid peroxidation in healthy humans. Free Radic Biol Med 37, 1351-1359.

10. Madrid AE, Vasques ZD, Leyton AF, et al. (2006) Short-term Lycopersicum esculentum consumption may increase plasma high density lipoproteins and decrease oxidative stress. Rev Med Chil 134, 855-862.

11. Gregory GK, Chen TS \& Philip T (1987) Quantitative analysis of catotenoids and carotenoid ester in fruits by HPLC: red bell peppers. J Food Sci 52, 1071-1073.

12. Biacs PA, Daood HG, Pavisa A, et al. (1989) Studies on the carotenoid pigments of paprika (Capsicum anпиит L. var Sz-20). J Agric Food Chem 37, 350-353.

13. Matsufuji H, Nakamura H, Chino M, et al. (1998) Antioxidant activity of capsanthin and the fatty acid ester in paprika (Capsicum annuum). J Agric Food Chem 46, 3468-3472.

14. Oshima S, Sakamoto H, Ishiguro Y, et al. (1997) Accumulation and clearance of capsanthin in blood plasma after ingestion of paprika juice in men. $J$ Nutr 127, 1475-1479.

15. Boey PL, Nagao A, Terao J, et al. (1992) Antioxidant activity of xanthophylls on peroxyl radical-mediated phospholipids peroxidation. Biochim Biophs Acta 1126, 178-184.

16. Ojima F, Sakamoto H, Ishiguro Y, et al. (1993) Consumption of carotenoids in photosensitized oxidation of human plasma and plasma low-density lipoprotein. Free Radical Biol Med 15, 377-384. 
17. Brady WE, Mares-Perlman JA, Bowen P, et al. (1996) Human serum carotenoid concentrations are related to physiologic and lifestyle factors. J Nutr 126, 129-137.

18. Slattery ML, Jacobs DR Jr, Dyer A, et al. (1995) Dietary antioxidants and plasma lipids: the CARDIA Study. $J \mathrm{Am}$ Coll Nutr 14, 635-642.

19. Sugiura M, Matsumoto H, Kato M, et al. (2004) Seasonal changes in the relationship between serum concentration of $\beta$-cryptoxanthin and serum lipid levels. J Nutr Sci Vitaminol (Tokyo) 50, 410-415.

20. Hussein G, Nakagawa T, Goto H, et al. (2007) Astaxanthin ameliorates features of metabolic syndrome in SHR/NDmcr-cp. Life Sci 80, 522-529.

21. Jeyakumar SM, Vajreswari A \& Giridharan NV (2007) Impact of vitamin A on high-density lipoprotein-cholesterol and scavenger receptor class BI in the obese rat. Obesity (Silver Spring) 15, 322-329.

22. Association of Analytical Communities (1980) Vitamins and other nutrient/carotenes and xanthophylls in dried plant materials and mixed feeds. In Official Methods of Analysis, 13th ed., pp. 734-739 [W Horwitz and GW Latimer Jr, editors]. Washington, DC: AOAC International.

23. Aizawa K \& Inakuma T (2007) Quantitation of carotenoids in commonly consumed vegetables in Japan. Food Sci Technol Res 13, 247-252.

24. Sugiyama K, Yamakawa A, Kawagishi H, et al. (1997) Dietary eritadenine modifies plasma phosphatidylcholine molecular species profile in rats fed different types of fat. $J$ Nutr 127, 593-599.

25. Shimada Y, Morita T \& Sugiyama K (2003) Eritadenineinduced alterations of plasma lipoprotein lipid concentrations and phosphatidylcholine molecular species profile in rats fed cholesterol-free and cholesterol-enriched diets. Biosci Biotechnol Biochem 67, 996-1006.

26. Shimada Y, Yamakawa A, Morita T, et al. (2003) Effects of dietary eritadenine on the liver microsomal $\Delta 6$-desaturase activity and its mRNA in rats. Biosci Biotechnol Biochem 67, $1258-1266$.

27. Ito Y, Kurabe T \& Ishiguchi T (2004) The protective effect of lycopene against radiation injury to the small intestine of abdominally radiated mice. Jpn J Clin Physiol 34, 5-16.

28. Narisawa T, Fukaura Y, Hasebe M, et al. (2000) Prevention of $\mathrm{N}$ methylnitrosourea-induced colon carcinogenesis in rats by oxygenated carotenoid capsanthin and capsanthin-rich paprika juice. Proc Soc Exp Biol Med 224, 116-122.

29. Haack VS, Chester JG, Vollendorf NW, et al. (1998) Increasing amount of dietary fiber provided by foods normalizes physiologic response of the large bowel without altering calcium balance or fecal steroid excretion. Am J Clin Nutr $\mathbf{6 8}$, 615-622.

30. Cummings JH (1993) The effect of dietary fiber on fecal weight and composition. In Dietary Fiber in Human Nutrition, 2nd ed., pp. 263-349 [GA Speiller, editor]. Boca Raton, FL: CRC Press.

31. Jenkins DJ, Kendall CW, Axelsen M, et al. (2000) Viscous and nonviscous fibres, nonabsorbable and low glycaemic index carbohydrates, blood lipids and coronary heart disease. Curr Opin Lipidol 11, 49-56.

32. Marlett JA (1997) Sites and mechanisms for the hypocholesterolemic actions of soluble dietary fiber sources. Adv Exp Med Biol 427, 109-121.

33. Gallaher DD (2006) Dietary fiber. In Present Knowledge in Nutrition, 9th ed., pp. 102-110 [BA Bowman and RM Russell, editors]. Washington, DC: ILSI Press.

34. Zannis VI, Chroni A, Kypreos KE, et al. (2004) Probing the pathways of chylomicron and HDL metabolism using adenovirus-mediated gene transfer. Curr Opin Lipidol 15, $151-166$.

35. Krieger M (2001) Scavenger receptor class B type $I$ is a multiligand HDL receptor that influences diverse physiologic systems. J Clin Invest 108, 793-797.

36. Hoeg JM, Santamarina-Fojo S, Bérard AM, et al. (1996) Overexpression of lecithin:cholesterol acyltransferase in transgenic rabbits prevents diet-induced atherosclerosis. Proc Natl Acad Sci U S A 93, 11448-11453.

37. Brousseau ME, Santamarina-Fojo S, Vaisman BL, et al. (1997) Overexpression of human lecithin:cholesterol acyltransferase in cholesterol-fed rabbits: LDL metabolism and HDL metabolism are affected in a gene dose-dependent manner. J Lipid Res 38, $2537-2547$

38. Mehlum A, Staels B, Duverger N, et al. (1995) Tissue-specific expression of the human gene for lecithin:cholesterol acyltransferase in transgenic mice alters blood lipids, lipoproteins and lipases towards a less atherogenic profile. Eur J Biochem 230, $567-575$.

39. Ettinger WH, Miller LD, Albers JJ, et al. (1990) Lipopolysaccharide and tumor necrosis factor cause a fall in plasma concentration of lecithin:cholesterol acyltransferase in cynomolgus monkeys. J Lipid Res 31, 1099-1107.

40. Auerbach BJ \& Parks JS (1989) Lipoprotein abnormalities associated with lipopolysaccharide-induced lecithin:cholesterol acyltransferase and lipase deficiency. J Biol Chem 264, $10264-10270$.

41. Forte TM, Oda MN, Knoff L, et al. (1999) Targeted disruption of the murine lecithin:cholesterol acyltransferase gene is associated with reductions in plasma paraoxonase and plateletactivating factor acetylhydrolase activities but not in apolipoprotein J concentration. J Lipid Res 40, 1276-1283.

42. Pennacchio LA, Olivier M, Hubacek JA, et al. (2001) An apolipoprotein influencing triglycerides in humans and mice revealed by comparative sequencing. Science 294, 169-173.

43. van der Vliet HN, Sammels MG, Leegwater AC, et al. (2001) Apolipoprotein A-V: a novel apolipoprotein associated with an early phase of liver regeneration. J Biol Chem 276, 44512-44520.

44. O'Brien PJ, Alborn WE, Sloan JH, et al. (2005) The novel apolipoprotein A5 is present in human serum, is associated with VLDL, HDL, and chylomicrons, and circulates at very low concentrations compared with other apolipoproteins. Clin Chem 51, 351-359.

45. Pennacchio LA \& Rubin EM (2003) Apolipoprotein A5, a newly identified gene that affects plasma triglyceride levels in humans and mice. Arterioscler Thromb Vasc Biol 23, 529-534.

46. Marçais C, Verges B, Charrière S, et al. (2005) ApoA5 Q139X truncation predisposes to late-onset hyperchylomicronemia due to lipoprotein lipase impairment. J Clin Invest 115, 2862-2869.

47. Qu S, Perdomo G, Su D, et al. (2007) Effects of apoA-V on HDL and VLDL metabolism in APOC3 transgenic mice. $J$ Lipid Res 48, 1476-1487.

48. Sluijs I, Beulens JW, Grobbee DE, et al. (2009) Dietary carotenoid intake is associated with lower prevalence of metabolic syndrome in middle-aged and elderly men. J Nutr 139, 987-992.

49. Waters D, Clark RM, Greene CM, et al. (2007) Change in plasma lutein after egg consumption is positively associated with plasma cholesterol and lipoprotein size but negatively correlated with body size in postmenopausal women. $J$ Nutr 137, 959-963. 